\title{
Existence of Solutions for Optimal Control Problems on Time Scales whose States are Absolutely Continuous
}

\author{
I.L.D. SANTOS
}

Received on June 21, 2015 / Accepted on February 17, 2016

\begin{abstract}
This paper considers a class of optimal control problems on time scales described by dynamic equations on time scales. For this class, the states are absolutely continuous. We have established sufficient conditions for the existence of optimal controls.
\end{abstract}

Keywords: Time scales, control systems, optimal control.

\section{INTRODUCTION}

The existence of solutions to optimal control problems on time scales can be found in $[7,11,13]$. In this report, we have studied control systems covered in [6] to the study of necessary optimality conditions. However, unlike [6] here the states are absolutely continuous functions and the controls are $\Delta$-measurable functions. To the class of control systems studied here, we establish an extension of Filippov's selection theorem [4].

Using standards arguments to the obtaining of optimal controls, see for instance $[4,8,12]$, we get the existence of solutions to optimal control problems on time scales. The result of existence obtained here relies on [[11], Theorem 5.2], the difference is in the classes of control systems studied.

The paper is organized as follows. In Section 2, we provided basic concepts and results on time scales theory. Section 3 presents the result of existence of optimal processes to optimal control problems on time scales.

\section{PRELIMINARIES}

\subsection{Time scales}

A time scale is a nonempty closed subset of real numbers. Here we use a bounded time scale $\mathbb{T}$, where $a=\min \mathbb{T}$ and $b=\max \mathbb{T}$ are such that $a<b$.

Departamento de Matemática - FEIS, UNESP - Universidade Estadual Paulista, 15385-000 Ilha Solteira, SP, Brasil. E-mail: iguerluis@mat.feis.unesp.br 
Define the forward jump operator $\sigma: \mathbb{T} \rightarrow \mathbb{T}$ by

$$
\sigma(t)=\inf \{s \in \mathbb{T}: s>t\}
$$

and the backward jump operator $\rho: \mathbb{T} \rightarrow \mathbb{T}$ by

$$
\rho(t)=\sup \{s \in \mathbb{T}: s<t\} .
$$

We assume that $\inf \emptyset=\sup \mathbb{T}$ and $\sup \emptyset=\inf \mathbb{T}$.

Let $\mu: \mathbb{T} \rightarrow[0,+\infty)$ be defined as

$$
\mu(t)=\sigma(t)-t
$$

Lemma 2.1 ([3]). There exist $I \subset \mathbb{N}$ and $\left\{t_{i}\right\}_{i \in I} \subset \mathbb{T}$ such that

$$
R S:=\{t \in \mathbb{T}: t<\sigma(t)\}=\left\{t_{i}\right\}_{i \in I},
$$

where $R S$ stands for right scattered points of the time scale $\mathbb{T}$.

If $A \subset \mathbb{R}$, define the set $A_{\mathbb{T}}$ as $A_{\mathbb{T}}=A \cap \mathbb{T}$. We set $\mathbb{T}^{\kappa}=\mathbb{T} \backslash(\rho(\sup \mathbb{T}) \text {, sup } \mathbb{T}]_{\mathbb{T}}$.

Take a function $f: \mathbb{T} \rightarrow \mathbb{R}$ and $t \in \mathbb{T}^{\kappa}$. If $\xi \in \mathbb{R}$ is such that, for all $\varepsilon>0$ there exists $\delta>0$ satisfying

$$
|f(\sigma(t))-f(s)-\xi(\sigma(t)-s)| \leq \varepsilon|\sigma(t)-s|
$$

for all $s \in(t-\delta, t+\delta)_{\mathbb{T}}$, we say that $\xi$ is the delta derivative of $f$ at $t$ and we denote it by $\xi:=f^{\Delta}(t)$.

Now, consider a function $f: \mathbb{T} \rightarrow \mathbb{R}^{n}$ and $t \in \mathbb{T}^{\kappa}$. We say that $f$ is $\Delta$-differentiable at $t$ if each component $f_{i}: \mathbb{T} \rightarrow \mathbb{R}$ of $f$ is $\Delta$-differentiable at $t$. In this case $f^{\Delta}(t)=\left(f_{1}^{\Delta}(t), \ldots, f_{n}^{\Delta}(t)\right)$.

The next result is an easy consequence of [[1], Theorem 1.16].

Theorem 2.1 ([1]). Consider a function $f: \mathbb{T} \rightarrow \mathbb{R}^{n}$ and $t \in \mathbb{T}^{\kappa}$. Then the following statements hold:

(i) If $f$ is $\Delta$-differentiable at $t$ then $f$ is continuous at $t$.

(ii) If $f$ is continuous at $t$ and $\sigma(t)>t$, then $f$ is $\Delta$-differentiable at $t$. Furthermore,

$$
f^{\Delta}(t)=\frac{f(\sigma(t))-f(t)}{\mu(t)} .
$$

(iii) If $\sigma(t)=t$, then $f$ is $\Delta$-differentiable at $t$ if and only if the following limit exists

$$
\lim _{s \underset{\mathbb{T}}{\rightarrow} t} \frac{f(t)-f(s)}{t-s}
$$

as an element of $\mathbb{R}^{n}$. In this case

$$
f^{\Delta}(t)=\lim _{s \underset{\mathbb{T}}{\rightarrow} t} \frac{f(t)-f(s)}{t-s} .
$$


(iv) If $f$ is $\Delta$-differentiable at $t$, then

$$
f(\sigma(t))=f(t)+\mu(t) f^{\Delta}(t)
$$

\section{2 $\Delta$-measurable functions and $\Delta$-integrability}

We denote the family of $\Delta$-measurable sets of $\mathbb{T}$ by $\Delta$. We recall that $\Delta$ is a $\sigma$-algebra of $\mathbb{T}$.

Given a function $f: \mathbb{T} \rightarrow \mathbb{R}^{n}$ we define $\tilde{f}:[a, b] \rightarrow \mathbb{R}^{n}$ as

$$
\tilde{f}(t)=\left\{\begin{array}{l}
f(t), t \in \mathbb{T} \\
f\left(t_{i}\right), \quad t \in\left(t_{i}, \sigma\left(t_{i}\right)\right) \text { for some } i \in I
\end{array}\right.
$$

where $I \subset \mathbb{N}$ and $\left\{t_{i}\right\}_{i \in I} \subset \mathbb{T}$ are such that $\{t \in \mathbb{T}: t<\sigma(t)\}=\left\{t_{i}\right\}_{i \in I}$.

Proposition 2.1 given below can be easily obtained from [[3], Proposition 4.1].

Proposition 2.1 ([3]). Consider a function $f: \mathbb{T} \rightarrow \mathbb{R}^{n}$. Then $f$ is $\Delta$-measurable if and only if $\tilde{f}$ is Lebesgue measurable.

Take a function $f: \mathbb{T} \rightarrow \overline{\mathbb{R}}$ and $E \in \Delta$. We indicate by

$$
\int_{E} f(s) \Delta s
$$

the Lebesgue $\Delta$-integral of $f$ over $E$. We denote the set of functions $f: \mathbb{T} \rightarrow \mathbb{R}$ which are Lebesgue $\Delta$-integrable over $E$ by $L_{1}(E)$. If $f: \mathbb{T} \rightarrow \mathbb{R}^{n}$ is $\Delta$-measurable and $E \in \Delta$, we indicate by $L_{1}\left(E, \mathbb{R}^{n}\right)$ the set of functions $f: \mathbb{T} \rightarrow \mathbb{R}^{n}$ which are Lebesgue $\Delta$-integrable over $E$.

More in the subject on Lebesgue integration on time scales, can be found in $[3,5,10]$.

Proposition 2.2 ([10]). Let $f: \mathbb{T} \rightarrow[0,+\infty)$ be a function in $L_{1}\left([a, b)_{\mathbb{T}}\right)$. Given $\varepsilon>0$ there exists $\delta>0$ such that, if $A \in \Delta$ and $\mu_{\Delta}(A)<\delta$ then

$$
\int_{A} f(s) \Delta s<\varepsilon .
$$

Proposition 2.3 ([10]). Let $g \in L_{1}\left([a, b)_{\mathbb{T}}\right)$. Suppose that

$$
\int_{[c, d)_{\mathbb{T}}} g(s) \Delta s \geq 0
$$

for each $c, d \in \mathbb{T}$ such that $c<d$. Then $g(t) \geq 0 \Delta$-a.e. $t \in[a, b)_{\mathbb{T}}$.

\subsection{Absolutely continuous functions on time scales}

A function $f: \mathbb{T} \rightarrow \mathbb{R}^{n}$ is absolutely continuous if given $\varepsilon>0$ there exists $\delta>0$ such that

$$
\sum_{i=1}^{N}\left\|f\left(b_{i}\right)-f\left(a_{i}\right)\right\|<\varepsilon
$$


whenever $a_{i} \leq b_{i}$ and $\left\{\left[a_{i}, b_{i}\right)_{\mathbb{T}}\right\}_{i=1}^{N}$ are disjoint intervals satisfying

$$
\sum_{i=1}^{N}\left(b_{i}-a_{i}\right)<\delta .
$$

The next theorem is an immediate consequence of [[2], Theorem 4.1].

Theorem 2.2. A function $f: \mathbb{T} \rightarrow \mathbb{R}^{n}$ is absolutely continuous if and only if the following assertions are valid:

1. $f$ is $\Delta$-differentiable $\Delta$-a.e. on $[a, b)_{\mathbb{T}}$ and $f^{\Delta} \in L_{1}\left([a, b)_{\mathbb{T}}, \mathbb{R}^{n}\right)$;

2. for each $t \in \mathbb{T}$ we have

$$
f(t)=f(a)+\int_{[a, t)_{\mathbb{T}}} f^{\Delta}(s) \Delta s
$$

A function $f: \mathbb{T} \rightarrow \mathbb{R}^{n}$ is said to be an arc if it is absolutely continuous. We denote the set of all arcs with domain $\mathbb{T}$ and taking values on $\mathbb{R}^{n}$ by $A C\left(\mathbb{T}, \mathbb{R}^{n}\right)$.

Proposition 2.4 ([10]). Consider the sequence $x_{i}: \mathbb{T} \rightarrow \mathbb{R}^{n}$ of arcs and a function $\phi: \mathbb{T} \rightarrow$ $[0,+\infty)$ in $L_{1}\left([a, b)_{\mathbb{T}}\right)$. Suppose that for each $i$, we have

$$
\left\|x_{i}^{\Delta}(s)\right\| \leq \phi(s) \quad \Delta-\text { a.e. } s \in[a, b)_{\mathbb{T}} .
$$

If $\left\{x_{i}(a)\right\}$ is a bounded sequence, then there exists a subsequence $\left\{x_{i_{k}}\right\} \subset\left\{x_{i}\right\}$ and an arc $x$ : $\mathbb{T} \rightarrow \mathbb{R}^{n}$ such that $x_{i_{k}} \rightrightarrows x$. Furthermore, if $c, d \in \mathbb{T}$ and $c<d$ then

$$
\int_{[c, d)_{\mathbb{T}}} x_{i_{k}}^{\Delta}(s) \Delta s \rightarrow \int_{[c, d)_{\mathbb{T}}} x^{\Delta}(s) \Delta s .
$$

Proposition 2.5 ([10]). Let $x: \mathbb{T} \rightarrow \mathbb{R}^{n}$ be an arc and let $k: \mathbb{T} \rightarrow[0,+\infty)$ be a function in $L_{1}\left([a, b)_{\mathbb{T}}\right)$. Suppose

$$
\left\|x^{\Delta}(t)\right\| \leq \gamma_{1}\|x(t)\|+k(t) \quad \Delta-\text { a.e. } t \in[a, b)_{\mathbb{T}}
$$

with $\gamma_{1} \geq 0$. Then

a) $\|x(t)-x(a)\| \leq \gamma_{1}\|x(a)\| \int_{[a, t)_{\mathbb{T}}} e^{\gamma_{1}(t-s)} \Delta s+\int_{[a, t)_{\mathbb{T}}} k(s) e^{\gamma_{1}(t-s)} \Delta s$; and

b) $\|x(t)\| \leq\left(\gamma_{1} e^{\gamma_{1}(b-a)}(b-a)+1\right)\|x(a)\|+e^{\gamma_{1}(b-a)} \int_{[a, b)_{\mathbb{T}}} k(s) \Delta s$

for all $t \in \mathbb{T}$. 


\subsection{Set-valued functions properties}

Here we consider basic results of measurable set-valued functions.

Let $(\Omega, \mathcal{F})$ be a measurable space. A set-valued function $E: \Omega \rightsquigarrow \mathbb{R}^{n}$ is said to be $\mathcal{F}$-measurable if the set

$$
E^{-1}(V)=\{x \in \Omega: E(x) \cap V \neq \emptyset\}
$$

is $\mathcal{F}$-measurable for all compact sets $V \subset \mathbb{R}^{n}$.

A set-valued function $E$ is said to be closed, compact, convex or nonempty when its image $E(x)$ obeys the required property, each point $x \in \Omega$.

Definition 2.1. Take $U: \mathbb{T} \rightsquigarrow \mathbb{R}^{m}$. We define the set-valued function $\tilde{U}:[a, b] \rightsquigarrow \mathbb{R}^{m}$ by

$$
\tilde{U}(t)=\left\{\begin{array}{l}
U(t), t \in \mathbb{T} \\
U\left(t_{i}\right), \quad t \in\left(t_{i}, \sigma\left(t_{i}\right)\right) \text { for some } i \in I .
\end{array}\right.
$$

Lemma 2.2 ([11]). Let $U: \mathbb{T} \rightsquigarrow \mathbb{R}^{m}$ be a $\Delta$-measurable set-valued function. Then the set-valued function $\tilde{U}:[a, b] \rightsquigarrow \mathbb{R}^{m}$ is Lebesgue measurable.

Similarly to [[12], Theorem 2.3.14], we have the proposition below.

Proposition 2.6. Consider a function $g:[a, b] \times \mathbb{R}^{m} \rightarrow \mathbb{R}$ and a closed nonempty set-valued function $\Gamma:[a, b] \rightsquigarrow \mathbb{R}^{m}$. Assume that

(a) $g$ is a Carathéodory function;

(b) $\Gamma$ is a Lebesgue measurable set-valued function.

Let $\eta:[a, b] \rightarrow \overline{\mathbb{R}}$ be defined by

$$
\eta(t)=\sup _{u \in \Gamma(t)} g(t, u)
$$

Then $\eta$ is a Lebesgue measurable function.

Using Proposition 2.1 and Lemma 2.2, we get the following consequence of the Proposition 2.6.

Lemma 2.3. Consider a function $g: \mathbb{T} \times \mathbb{R}^{m} \rightarrow \mathbb{R}$ and a closed nonempty set-valued function $U: \mathbb{T} \rightsquigarrow \mathbb{R}^{m}$. Assume that

(a) $g$ is $\Delta$-measurable at $t$, for each fixed $(x, u)$, and continuous at $(x, u)$, for each fixed $t$;

(b) $U$ is a $\Delta$-measurable set-valued function. 
Then the function $\eta: \mathbb{T} \rightarrow \overline{\mathbb{R}}$ given by

$$
\eta(t)=\sup _{u \in U(t)} g(t, u)
$$

is a $\Delta$-measurable function.

Take a function $f: \mathbb{T} \times \mathbb{R}^{n} \times \mathbb{R}^{m} \rightarrow \mathbb{R}^{n}$ and a set-valued function $U: \mathbb{T} \rightsquigarrow \mathbb{R}^{m}$. Consider the following hypotheses:

(H1) $f$ is continuous at $(x, u)$, for each fixed $t$, and $\Delta$-measurable at $t$, for each fixed $(x, u)$.

(H2) $U$ is a compact, nonempty and $\Delta$-measurable set-valued function.

We define the function $H: \mathbb{T} \times \mathbb{R}^{n} \times \mathbb{R}^{n} \rightarrow \mathbb{R}$ as

$$
H(t, x, p)=\max _{u \in U(t)}\langle p, f(t, x, u)\rangle
$$

If $x: \mathbb{T} \rightarrow \mathbb{R}^{n}$ is a $\Delta$-measurable function and $p \in \mathbb{R}^{n}$, it is a consequence of Lemma 2.3 that

$$
t \mapsto H(t, x(t), p)
$$

is a $\Delta$-measurable function.

\subsection{Filippov's selection theorem}

Consider the control system of equations

$$
\left\{\begin{array}{l}
x^{\Delta}(t)=f(t, x(\sigma(t)), u(t)) \quad \Delta-\text { a.e. } t \in[a, b)_{\mathbb{T}} \\
u(t) \in U(t) \Delta-\text { a.e. } t \in[a, b)_{\mathbb{T}} .
\end{array}\right.
$$

If the control process pair $(x, u)$ obeys $(2.1)$ then $x$ obeys the following dynamical inclusion

$$
x^{\Delta}(t) \in\{f(t, x(\sigma(t)), u): u \in U(t)\} \quad \Delta-\text { a.e. } t \in[a, b)_{\mathbb{T}} .
$$

Similar to [[11],Theorem 4.8] we have the following extension of Filippov's selection theorem.

Theorem 2.3. Consider a function $f: \mathbb{T} \times \mathbb{R}^{n} \times \mathbb{R}^{m} \rightarrow \mathbb{R}^{n}$ and a set-valued function $U: \mathbb{T} \rightsquigarrow$ $\mathbb{R}^{m}$. Assume that the function $f$ and the set-valued function $U$ satisfy the hypothesis (H1) and (H2).

If $x \in A C\left(\mathbb{T}, \mathbb{R}^{n}\right)$ obeys (2.2) then there is a $\Delta$-measurable selection $u$ of $U$ such that the process $(x, u)$ obeys $(2.1)$. 


\section{OPTIMAL CONTROL PROBLEMS IN TIME SCALES}

Here we get the existence of optimal controls to optimal control problems on time scales.

Consider the optimal control problem on time scales

$$
(P)\left\{\begin{array}{l}
\min g(x(a), x(b)) \text { over }(x, u) \text { obeying } \\
x^{\Delta}(t)=f(t, x(\sigma(t)), u(t)) \quad \Delta-\text { a.e. } t \in[a, b)_{\mathbb{T}} \\
u(t) \in U(t) \quad \Delta-\text { a.e. } t \in[a, b)_{\mathbb{T}} \\
(x(a), x(b)) \in A \times C
\end{array}\right.
$$

where $A, C \subset \mathbb{R}^{n}, x \in A C\left(\mathbb{T}, \mathbb{R}^{n}\right), u: \mathbb{T} \rightarrow \mathbb{R}^{m}$ is a $\Delta$-measurable function, $g: \mathbb{R}^{n} \times \mathbb{R}^{n} \rightarrow \mathbb{R}$, $f: \mathbb{T} \times \mathbb{R}^{n} \times \mathbb{R}^{m} \rightarrow \mathbb{R}^{n}$ and $U: \mathbb{T} \rightsquigarrow \mathbb{R}^{m}$ is a set-valued function.

Consider the following hypotheses:

(H3) $A$ is a compact set and $C$ is a closed set.

(H4) $g$ is a lower semicontinuous function.

(H5) There exists $K>0$ such that for any $t \in \mathbb{T}$, one has

$$
\|f(t, x, u)-f(t, y, u)\| \leq K\|x-y\|
$$

for any $u \in U(t)$, and any $x, y \in \mathbb{R}^{n}$.

(H6) There are positive constant $\gamma>0$ and a function $c: \mathbb{T} \rightarrow[0, \infty)$ in $L_{1}\left([a, b)_{\mathbb{T}}\right)$ such that

$$
\|f(t, x, u)\| \leq \gamma\|x\|+c(t) \quad \Delta-\text { a.e. } t \in[a, b)_{\mathbb{T}}
$$

for all $x \in \mathbb{R}^{n}$ and all $u \in U(t)$.

(H7) The set $f(t, x, U(t))$ is convex for each $t \in \mathbb{T}$ and $x \in \mathbb{R}^{n}$.

(H8) Let $j>0$. Suppose that

$$
\gamma \mu(t)<1 \quad \forall t \in \mathbb{T}
$$

and

$$
\frac{1}{1-\gamma \mu(t)} \leq j \quad \forall t \in \mathbb{T}
$$

Let $u: \mathbb{T} \rightarrow \mathbb{R}^{m}$ be a $\Delta$-measurable function and $x \in A C\left(\mathbb{T}, \mathbb{R}^{n}\right)$. We say that $(x, u)$ is an admissible process for $(P)$, if the pair $(x, u)$ satisfies $(2.1)$ and $x$ obeys the condition $(x(a), x(b)) \in$ $A \times C$. A process $(\bar{x}, \bar{u})$ is called an optimal process for $(P)$, if it is an admissible process for $(P)$ that satisfies

$$
g(\bar{x}(a), \bar{x}(b)) \leq g(x(a), x(b))
$$


for all admissible process $(x, u)$ of $(P)$.

Theorem 3.4 ensures the existence of optimal processes for $(P)$. We get the Theorem 3.4 using the next two lemmas.

Lemma 3.4. Assume (H8). Let $f: \mathbb{T} \times \mathbb{R}^{n} \times \mathbb{R}^{m} \rightarrow \mathbb{R}^{n}$ be a function obeying (H6). If $(x, u)$ satisfies (2.1) then

$$
\left\|x^{\Delta}(t)\right\| \leq \frac{\gamma}{1-\gamma \mu(t)}\|x(t)\|+\left(1+\frac{\gamma \mu(t)}{1-\gamma \mu(t)}\right) c(t) \quad \Delta-a . e . t \in[a, b)_{\mathbb{T}} .
$$

Proof. Let $t \in[a, b)_{\mathbb{T}}$ be such that

$$
x^{\Delta}(t)=f(t, x(\sigma(t)), u(t))
$$

If $\sigma(t)=t$ we have

$$
\begin{aligned}
\left\|x^{\Delta}(t)\right\| & =\|f(t, x(t), u(t))\| \leq \gamma\|x(t)\|+c(t) \\
& \leq \frac{\gamma}{1-\gamma \mu(t)}\|x(t)\|+\left(1+\frac{\gamma \mu(t)}{1-\gamma \mu(t)}\right) c(t)
\end{aligned}
$$

and if $\sigma(t)>t$ we get

$$
\begin{aligned}
\left\|x^{\Delta}(t)\right\| & =\|f(t, x(\sigma(t)), u(t))\| \leq \gamma\|x(\sigma(t))\|+c(t) \\
& \leq \frac{\gamma}{1-\gamma \mu(t)}\|x(t)\|+\left(1+\frac{\gamma \mu(t)}{1-\gamma \mu(t)}\right) c(t)
\end{aligned}
$$

since

$$
\begin{aligned}
\left\|x^{\Delta}(t)\right\| & =\left\|\frac{x(\sigma(t))-x(t)}{\mu(t)}\right\|=\|f(t, x(\sigma(t)), u(t))\| \\
& \leq \gamma\|x(\sigma(t))\|+c(t)
\end{aligned}
$$

and

$$
\frac{\|x(\sigma(t))\|-\|x(t)\|}{\mu(t)} \leq\left\|\frac{x(\sigma(t))-x(t)}{\mu(t)}\right\|
$$

and then

$$
\|x(\sigma(t))\| \leq \frac{1}{1-\gamma \mu(t)}\|x(t)\|+\frac{\mu(t) c(t)}{1-\gamma \mu(t)} .
$$

Therefore

$$
\left\|x^{\Delta}(t)\right\| \leq \frac{\gamma}{1-\gamma \mu(t)}\|x(t)\|+\left(1+\frac{\gamma \mu(t)}{1-\gamma \mu(t)}\right) c(t) \quad \Delta-\text { a.e. } t \in[a, b)_{\mathbb{T}} .
$$


Lemma 3.5. Assume (H8) and consider a function $f: \mathbb{T} \times \mathbb{R}^{n} \times \mathbb{R}^{m} \rightarrow \mathbb{R}^{n}$ obeying (H6). Take a sequence $\left(x_{i}, u_{i}\right)$ satisfying (2.1). If $\left\{x_{i}(a)\right\}$ is a bounded sequence, then there exists a subsequence $\left\{x_{i_{k}}\right\} \subset\left\{x_{i}\right\}$ and an arc $x: \mathbb{T} \rightarrow \mathbb{R}^{n}$ such that $x_{i_{k}} \rightrightarrows x$.

Proof. Since

$$
1 \leq \frac{1}{1-\gamma \mu(t)} \leq j \quad \forall t \in \mathbb{T}
$$

it follows from Lemma 3.4 that

$$
\left\|x_{i}^{\Delta}(t)\right\| \leq j \gamma\left\|x_{i}(t)\right\|+(1+\gamma(b-a) j) c(t) \quad \Delta-a . e . t \in[a, b)_{\mathbb{T}} .
$$

Let $\gamma_{1}:=j \gamma$ and let $k(t):=(1+\gamma(b-a) j) c(t)$. Consider a real number $L>0$ obeying

$$
\left\|x_{i}(a)\right\| \leq L \quad \forall i
$$

From Proposition 2.5 we get

$$
\left\|x_{i}(t)\right\| \leq\left(\gamma_{1} e^{\gamma_{1}(b-a)}(b-a)+1\right) L+e^{\gamma_{1}(b-a)} \int_{[a, b)_{\mathbb{T}}} k(s) \Delta s:=L_{1}
$$

for all $t \in \mathbb{T}$. Then

$$
\left\|x_{i}^{\Delta}(t)\right\| \leq j \gamma L_{1}+k(t) \quad \Delta-\text { a.e. } t \in[a, b)_{\mathbb{T}} .
$$

Now, using Proposition 2.4 we conclude the proof.

Theorem 3.4. Assume that $(H 1)-(H 8)$ are satisfied for the optimal control problem $(P)$. If the problem $(P)$ has an admissible process, then there exists an optimal process $(x, u)$ for $(P)$.

Proof. Let $\inf \{P\}$ denote the greatest lower bound on $g(x(a), x(b))$ over admissible processes $(x, u)$ of $(P)$. Thence there exists a sequence of admissible processes $\left(x_{i}, u_{i}\right)$ of $(P)$ obeying

$$
\inf \{P\}=\lim _{i \rightarrow \infty} g\left(x_{i}(a), x_{i}(b)\right) .
$$

From Lemma 3.5 there exists a subsequence of $\left\{x_{i}\right\}$, we do not relabel, such that $\left\{x_{i}\right\}$ converges uniformly for an arc $x$, that is, $x_{i} \rightrightarrows x$.

Now we prove that there is a $\Delta$-measurable selection $u$ of $U$ such that $(x, u)$ is an admissible process for $(P)$.

Let $t, s \in \mathbb{T}$ be such that $t \leq s$. We have

$$
\begin{aligned}
& \lim _{i \rightarrow \infty} \int_{[t, s)_{\mathbb{T}}} f\left(\tau, x_{i}(\tau), u_{i}(\tau)\right) \Delta \tau \\
& =\lim _{i \rightarrow \infty}\left(x_{i}(s)-x_{i}(t)\right)=x(s)-x(t)=\int_{[t, s)_{\mathbb{T}}} x^{\Delta}(\tau) \Delta \tau
\end{aligned}
$$


By (H5) we get

$$
\begin{aligned}
& \left\|f\left(\tau, x_{i}(\tau), u_{i}(\tau)\right)-f\left(\tau, x(\tau), u_{i}(\tau)\right)\right\| \\
& \leq K\left\|x_{i}(\tau)-x(\tau)\right\| \quad \Delta-\text { a.e. } \tau \in[a, b)_{\mathbb{T}}
\end{aligned}
$$

and thus

$$
\lim _{i \rightarrow \infty}\left\|f\left(\tau, x_{i}(\tau), u_{i}(\tau)\right)-f\left(\tau, x(\tau), u_{i}(\tau)\right)\right\|=0 \quad \Delta-\text { a.e. } \tau \in[a, b)_{\mathbb{T}} .
$$

Note that

$$
\begin{aligned}
& \int_{[t, s)_{\mathbb{T}}}\left(f\left(\tau, x(\tau), u_{i}(\tau)\right)-x^{\Delta}(\tau)\right) \Delta \tau \\
& =\int_{[t, s)_{\mathbb{T}}}\left(f\left(\tau, x(\tau), u_{i}(\tau)\right)-f\left(\tau, x_{i}(\tau), u_{i}(\tau)\right)\right) \Delta \tau \\
& \quad+\int_{[t, s)_{\mathbb{T}}}\left(f\left(\tau, x_{i}(\tau), u_{i}(\tau)\right)-x^{\Delta}(\tau)\right) \Delta \tau
\end{aligned}
$$

and that

$$
\lim _{i \rightarrow \infty} \int_{[t, s)_{\mathbb{T}}}\left(f\left(\tau, x(\tau), u_{i}(\tau)\right)-f\left(\tau, x_{i}(\tau), u_{i}(\tau)\right)\right) \Delta \tau=0
$$

because of the dominated convergence theorem ([9]). Using Proposition 2.4, we obtain

$$
\begin{aligned}
& \lim _{i \rightarrow \infty} \int_{[t, s)_{\mathbb{T}}}\left(x_{i}^{\Delta}(\tau)-x^{\Delta}(\tau)\right) \Delta \tau \\
& =\lim _{i \rightarrow \infty} \int_{[t, s)_{\mathbb{T}}}\left(f\left(\tau, x_{i}(\tau), u_{i}(\tau)\right)-x^{\Delta}(\tau)\right) \Delta \tau=0 .
\end{aligned}
$$

Then

$$
\lim _{i \rightarrow \infty} \int_{[t, s)_{\mathbb{T}}}\left(f\left(\tau, x(\tau), u_{i}(\tau)\right)-x^{\Delta}(\tau)\right) \Delta \tau=0
$$

Let $p \in \mathbb{R}^{n}$ be arbitrarily fixed. Then by (3.1) we have

$$
\lim _{i \rightarrow \infty}\left\langle p, \int_{[t, s)_{\mathbb{T}}}\left(f\left(\tau, x(\tau), u_{i}(\tau)\right)-x^{\Delta}(\tau)\right) \Delta \tau\right\rangle=0 .
$$

For any $i$ we also have

$$
\begin{aligned}
& \int_{[t, s)_{\mathbb{T}}} \max _{u \in U(t)}\left\langle p,\left(f(\tau, x(\tau), u)-x^{\Delta}(\tau)\right)\right\rangle \Delta \tau \\
& \geq\left\langle p, \int_{[t, s)_{\mathbb{T}}}\left(f\left(\tau, x(\tau), u_{i}(\tau)\right)-x^{\Delta}(\tau)\right) \Delta \tau\right\rangle
\end{aligned}
$$


and therefore

$$
\int_{[t, s)_{\mathbb{T}}} \max _{u \in U(t)}\left\langle p,\left(f(\tau, x(\tau), u)-x^{\Delta}(\tau)\right)\right\rangle \Delta \tau \geq 0 .
$$

From Proposition 2.3 we deduce that

$$
\max _{v \in f(\tau, x(\tau), U(\tau))}\langle p, v\rangle \geq\left\langle p, x^{\Delta}(\tau)\right\rangle \quad \Delta-\text { a.e. } \tau \in[a, b)_{\mathbb{T}}
$$

that is,

$$
H(\tau, x(\tau), p) \geq\left\langle p, x^{\Delta}(\tau)\right\rangle \quad \Delta-\text { a.e. } \tau \in[a, b)_{\mathbb{T}} .
$$

As $p$ is arbitrary, we can use standard procedures (e.g., [12], p. 91) which involves the separability of $\mathbb{R}^{n}$ and the geometric Hahn-Banach separation theorem for convex sets, to obtain

$$
x^{\Delta}(t) \in\{f(t, x(\sigma(t)), u): u \in U(t)\} \quad \Delta-\text { a.e. } t \in[a, b)_{\mathbb{T}} .
$$

Theorem 2.3 ensures the existence of a $\Delta$-measurable selection $u$ of $U$ such that the process $(x, u)$ satisfies $(2.1)$.

Since $A \times C$ is a closed set we deduce that $(x(a), x(b)) \in A \times C$. Hence $(x, u)$ is an admissible process for $(P)$.

Finally, since

$$
\begin{aligned}
\inf \{P\} & =\lim _{i \rightarrow \infty} g\left(x_{i}(a), x_{i}(b)\right)=\liminf _{i \rightarrow \infty} g\left(x_{i}(a), x_{i}(b)\right) \\
& \geq g(x(a), x(b)) \geq \inf \{P\}
\end{aligned}
$$

we may conclude that $(x, u)$ is an optimal process for $(P)$.

RESUMO. Este artigo considera uma classe de problemas de controle ótimo em escalas temporais descritos por equações dinâmicas em escalas temporais. Para essa classe, os estados são absolutamente contínuos. Nós estabelecemos condições suficientes para a existência de controles ótimos.

Palavras-chave: escalas temporais, sistemas de controle, controle ótimo.

\section{REFERENCES}

[1] M. Bohner \& A. Peterson. "Dynamic Equations on Time Scales", Birkhäuser Boston Inc., Boston, (2001).

[2] A. Cabada \& D.R. Vivero. Criterions for absolute continuity on time scales, J. Difference Equ. Appl., 11(11) (2005), 1013-1028. 
[3] A. Cabada \& D.R. Vivero. Expression of the Lebesgue $\Delta$-integral on time scales as a usual Lebesgue integral: application to the calculus of $\Delta$-antiderivatives, Math. Comput. Modelling, 43(1-2) (2006), 194-207.

[4] A.F. Filippov. On certain questions in the theory of optimal control, J. SIAM Control Ser. A 1 (1962), 76-84.

[5] G.S. Guseinov. Integration on time scales, J. Math. Anal. Appl., 285(1) (2003), 107-127.

[6] R. Hilscher \& V. Zeidan. Weak maximum principle and accessory problem for control problems on time scales, Nonlinear Anal. 70(9) (2009), 3209-3226.

[7] Y. Peng, X. Xiang \& Y. Jiang. Nonlinear dynamic systems and optimal control problems on time scales, ESAIM Control Optim. Calc. Var., 17(3) (2011), 654-681.

[8] E. Roxin. The existence of optimal controls, Michigan Math. J., 9 (1962), 109-119.

[9] W. Rudin. "Real and Complex Analysis", third edition, McGraw-Hill Book Company, New York (1987).

[10] I.L.D. Santos \& G.N. Silva. Absolute continuity and existence of solutions to dynamic inclusions in time scales, Math. Ann., 356(1) (2013), 373-399.

[11] I.L.D. Santos \& G.N. Silva. Filippov's selection theorem and the existence of solutions for optimal control problems in time scales, Comput. Appl. Math., 33(1) (2014), 223-241.

[12] R.B. Vinter. "Optimal Control”, Systems and Control: Foundations and Applications, Birkhäuser, Boston (2000).

[13] Z. Zhan, W. Wei, Y. Li \& H. Xu. Existence for calculus of variations and optimal control problems on time scales, Int. J. Innov. Comput. Inf. Control, 8 (2012), 3793-3808. 